\title{
Joint Analysis of Longitudinal and Survival AIDS Data with a Spatial Fraction of Long- term Survivors: A Bayesian Approach
}

Rui Martins ${ }^{1}$, Giovani Silva ${ }^{2,4}$ \& Valeska Andreozzi ${ }^{3}$

\section{Overview}

The objective is to model longitudinal and survival data jointly taking into account the dependence between the two responses in a real HIV / AIDS dataset. We employ here a Bayesian hierarchical approach to jointly model spatialclustered survival data with a fraction of longterm survivors along with longitudinal measurements of $\mathrm{CD} 4^{+} \mathrm{T}$ lymphocyte counts for a random sample of $500 \mathrm{HIV}$ / AIDS individuals collected in all the 27 states of Brazil during the period 2002-2006.

In order to accommodate a more flexible choice for the longitudinal model we propose to use a specification via penalized B-Splines [2]. To deal with the possible fact that only a subpopulation undergoes the event we propose a spatial cure model, which helps in explaining the behavior of chronic or potentially terminal diseases in different regions. Classical survival models (e.g. Cox model) are not-well suited to take into account long-term survivors.

Results show that using a cure fraction allows us to improve the results comparatively to a survival analysis without it. The inclusion of spatial frailties eases in mapping the heterogeneity in the risk among the Brazilian states and helps in the explanation of the hazard.

\section{Dataset}

- Data origin: Brazilian database on HIV;

- Period (years): 2002-2006;

- Sample size: $n=500$ individuals

- Response variables: $y=\sqrt{\mathrm{CD}^{+} \mathrm{T}}$ lymphocyte counts and survival time (years since the patient's entry in the study until death)

- Explanatory variables: age $(<50=0, \geq 50=1)$; gender $($ Female $=0$, Male $=1)$; PrevOI (previous opportunistic infection at study entry $=1$, no previous infection $=0$ ); region of residence (one of the 27 Brazilian States)

- 34 deaths. $88 \%$ of the patients were between 15 and 49 years old; $60 \%$ were males. The CD4 counts initial median was 245 cells $/ \mathrm{mm}^{3}$ (men - 226 cells $/ \mathrm{mm}^{3}$; women 263 cells $/ \mathrm{mm}^{3}$ ).

\section{References}

[1] Cooner, F., Banerjee, S., and McBean, A.M. (2006) Modelling geographically referenced survival data with a cure fraction. Stat Meth Med Res, 15(4):307-324;

[2] Lang, S., Brezger, A. (2004) Bayesian p-splines. J Comp Graph Stat, 13(1):183-212;

[3] Martins, R., Silva, G.L., and Andreozzi, V. (2016) Bayesian joint modeling of longitudinal and spatial survival AIDS data. Stat Med, doi: 10.1002/sim.6937.

[4] Rizopoulos, D., Ghosh, P. (2011) A Bayesian semiparametric multivariate joint model for multiple longitudinal outcomes and a time-to-event. Stat Med, 30(12):1366-1380;

\section{Acknowledgments}

The authors wish to thank Maria Goretti Fonseca and Cláudia Coeli by the database. This work was partially funded by Fundação para a Ciência e a Tecnologia (FCT) projects PTDC/MAT/118335/2010 and UID/MAT/00006/2013 and Egas Moniz - Cooperativa de Ensino Superior, CRL

\section{Model}

Consider the CD4 repeated measurements, $\mathbf{y}_{i k}=\left(y_{i k 1}, \ldots, y_{i n_{i k}}\right)$, and the observed (possibly right censored) time-to-death, $T_{i k}$, for the $i$ th individual living in the $k$ th region, $k=1, \ldots, K$, $i=1, \ldots, n_{k}$. Longitudinal data is described by a nonlinear mixed effects model

$$
\begin{aligned}
y_{i k j} \mid \boldsymbol{b}_{i k}, \sigma_{e}^{2} & \sim \mathcal{N}\left(y_{i k}^{*}\left(t_{i k j}\right), \sigma_{e}^{2}\right), \quad j=1, \ldots, n_{i k}, \\
y_{i k}^{*}\left(t_{i k j}\right) & =\left(\beta_{10}+b_{i k 0}\right)+\mathbf{z}_{i k}^{\top} \boldsymbol{\beta}_{1}+m_{p}\left(t_{i k j} ; \boldsymbol{\beta}_{\mathbf{2}}\right)+m_{i k}\left(t_{i k j} ; \boldsymbol{b}_{i k}\right),
\end{aligned}
$$

where $m_{p}($.$) and m_{i k}($.$) are the overall and individual nonlinear effects, respectively, considered$ here as Penalized cubic B-Splines. $\beta_{10}$ and $b_{i k 0}$ are the common and subject-specific intercept, respectively. $\boldsymbol{\beta}_{1}=\left(\beta_{11}, \ldots, \beta_{1 r}\right)^{\top}$, whose elements are assumed to be mutually independent, represents the fixed effects of the subject-specific vector of covariates, $\mathbf{z}_{i k} ; \boldsymbol{\beta}_{2}=\left(\beta_{21}, \ldots, \beta_{2 Q}\right)^{\top}$ and $\boldsymbol{b}_{i k}=\left(b_{i k 1}, \ldots, b_{i k Q}\right)^{\top}$ are, respectively, population and individual-specific regression parameters for each basis function, penalized through a second order random-walk.

Assuming the time-to-death for the non-cured group is Weibull distributed, $\mathcal{W}\left(\rho, e^{\eta(t)}\right)$, the spatial cure model [1] is described as,

$$
S_{p}(t)=\left(1-\theta_{k}\right)+\theta_{k} S(t)=\left(1-\theta_{k}\right)+\theta_{k} \exp \left\{-t^{\rho} e^{\eta(t)}\right\},
$$

being $S_{p}$ and $S$ the survival functions for the entire population and for the non-cured group, respectively. $\left(1-\theta_{k}\right)$ is a region-specific cure fraction. The baseline covariates and the longitudinal information will be introduced through the scale parameter, $e^{\eta(t)}$, allowing it to vary across individuals and regions:

$$
\eta_{i k}(t)=\mathbf{x}_{i k}^{\top} \boldsymbol{\beta}_{3}+\gamma y_{i k}^{*}(t)+W_{k},
$$

where $\gamma$ is a parameter quantifying the effect of the CD4 values to the survival; $\mathbf{x}_{i k}$ is a vector of baseline covariates (can coincide with $\mathbf{z}_{i k}$ ), $\boldsymbol{\beta}_{3}$ is the respective vector of coefficients and $W_{k}$ is a region-specific frailty, $W_{k} \mid \sigma_{W}^{2} \sim \operatorname{ICAR}\left(\sigma_{W}^{2}\right)$.

\section{Application}

Several scenarios for the Penalized splines were tried. Namely the internal knots were fixed every: 1 month, 2 months, 3 months, 4 months, 6 months and 12 months providing, respectively, 59, 29, 19, 14,9 and 4 internal knots. The best fit was always achieved with 19 internal knots, corresponding roughly to a knot every 3 months during the 5 years, resulting in $Q=23$ cubic basis functions.

$$
\begin{aligned}
& y_{i k j}^{*}=\left(\beta_{10}+b_{i k 0}\right)+\beta_{11} \operatorname{sex}_{i k}+\beta_{12} \text { age }_{i k}+\beta_{13} \text { PrevOI }_{i k}+\sum_{q=1}^{Q=23} \beta_{2 q} B_{q}\left(t_{i k j}\right)+\sum_{q=1}^{Q=23} b_{i k q} B_{q}\left(t_{i k j}\right) \\
& \eta_{i k}(t)=\beta_{31}+\beta_{32} \text { sex }_{i k}+\beta_{33} \text { age }_{i k}+\beta_{34} \text { PrevOI }_{i k}+\gamma y_{i k}^{*}(t)+W_{k}
\end{aligned}
$$

\section{Results}

\begin{tabular}{ccc}
\hline $\boldsymbol{\pi}(\boldsymbol{\theta})$ & $\boldsymbol{W}_{\boldsymbol{k}}$ & Measure \\
\hline Common Cure & 0 & 14867 \\
$\theta_{k}=\theta, \forall k$ & & -7473 \\
$\theta \sim \mathcal{U}(0,1)$ & $W_{k}$ & $\mathbf{1 4 5 4 0 ( \mathrm { SM } )}$ \\
& & $-7453(\mathrm{SM})$ \\
Regional Cure & 0 & 14578 \\
$\theta_{k} \neq \theta_{l}, \forall k \neq l$ & & -7482 \\
$\theta_{k} \sim \mathcal{U}(0,1)$ & $W_{k}$ & 14596 \\
& & -7487 \\
No Cure & 0 & -74575 \\
$1-\theta_{k}=0, \forall k$ & & 14577 \\
& $W_{k}$ & -7496 \\
\hline
\end{tabular}

Model choice: Watanabe-Akaike Information Criterion (WAIC)

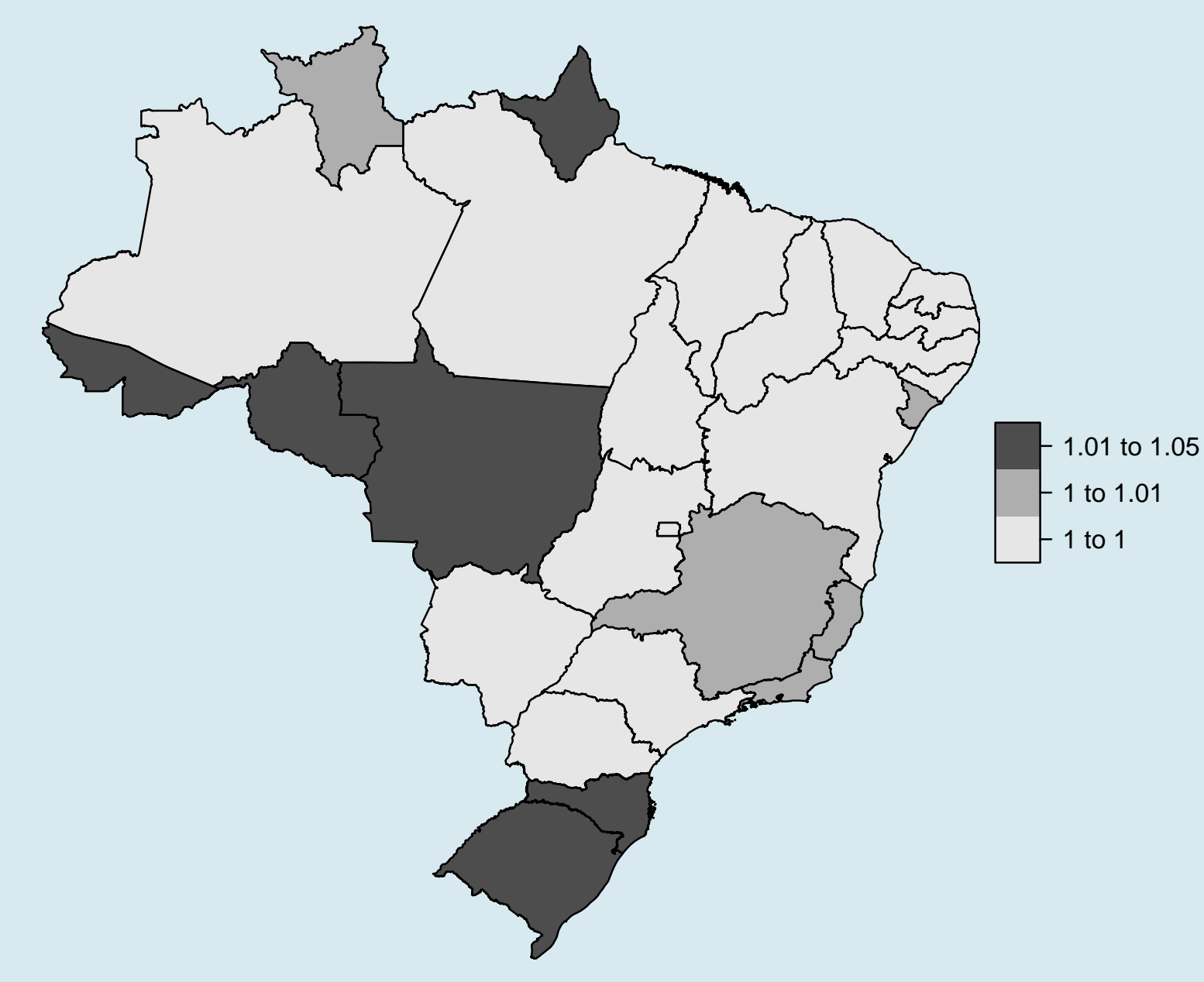

Spatial frailties - Brazil's map presenting the posterior median of the region-specific relative risks, $\exp \left\{W_{k}\right\}$

\begin{tabular}{lrr}
\hline & Mean & $\mathbf{9 5 \% ~ C I}$ \\
\hline$\left(\beta_{10}\right)$ & 14.65 & $(8.37,18.78)$ \\
$\left(\beta_{11}\right)$ sex & -0.15 & $(-2.66,2.08)$ \\
$\left(\beta_{12}\right)$ age & -1.30 & $(-4.25,1.30)$ \\
$\left(\beta_{13}\right)$ PrevOI & -1.84 & $(-3.67,-0.01)$ \\
$\sigma_{e}^{2}$ & 4.99 & $(4.54,5.26)$ \\
$\left(\beta_{31}\right)$ & -3.14 & $(-4.42,-1.87)$ \\
$\left(\beta_{32}\right)$ sex & 0.47 & $(-0.35,1.36)$ \\
$\left(\beta_{33}\right)$ age & 0.58 & $(-0.45,1.61)$ \\
$\left(\beta_{34}\right)$ PrevOI & 0.67 & $(-0.15,1.49)$ \\
$\gamma$ & -0.17 & $(-0.25,-0.11)$ \\
$\sigma_{W}^{2}$ & 0.015 & $(0.0002,0.13)$ \\
$\rho$ & 2.34 & $(1.67,3.11)$ \\
$1-\theta$ & 0.17 & $(0.01,0.47)$ \\
\hline
\end{tabular}

Parameters: posterior mean and its $95 \%$ Credibility Interval $(\mathrm{CI})$.

The model choice measures, WAIC and LPML agree in the the selected model (SM) $\Rightarrow$ Considering a common cure fraction for all patients and a spatial frailty outperforms the traditional joint model approach for the HIV / AIDS data, which does not considers a fraction of long-term survivors. The estimated cure fraction, $(1-\theta)$, indicates that nearly $17 \%$ of the HIV / AIDS patients in this study may be considered long-term survivors. 